\title{
Positive Solutions of a Kind of Equations Related to the Laplacian and $p$-Laplacian
}

\author{
Fangfang Zhang and Zhanping Liang \\ School of Mathematical Sciences, Shanxi University, Taiyuan, Shanxi 030006, China \\ Correspondence should be addressed to Zhanping Liang; lzp@sxu.edu.cn \\ Received 10 August 2014; Accepted 21 September 2014; Published 14 October 2014 \\ Academic Editor: P. Veeramani
}

Copyright (C) 2014 F. Zhang and Z. Liang. This is an open access article distributed under the Creative Commons Attribution License, which permits unrestricted use, distribution, and reproduction in any medium, provided the original work is properly cited.

Positive solutions of a kind of equations related to the Laplacian and $p$-Laplacian on a bounded domain in $\mathbb{R}^{N}$ with $N \geqslant 1$ are studied by using variational method. A sufficient condition of the existence of positive solutions is characterized by the eigenvalues of linear and another nonlinear eigenvalue problems.

\section{Introduction}

In this paper, we study the equation

$$
\begin{gathered}
-\Delta u-\Delta_{p} u=f(x, u), \quad \text { in } \Omega, \\
u=0, \quad \text { on } \partial \Omega,
\end{gathered}
$$

where $\Delta_{p} u=\operatorname{div}\left(|\nabla u|^{p-2} \nabla u\right), p>2, \Omega$ is a smooth bounded domain in $\mathbb{R}^{N}$ for $N \geqslant 1$, and $f$ satisfies the following conditions:

$\left(\mathrm{f}_{1}\right) f \in C(\bar{\Omega} \times \mathbb{R}, \mathbb{R}), f(x, t) \geqslant 0$ for any $x \in \bar{\Omega}, t>0$ and $f(x, t)=0$ for any $x \in \bar{\Omega}, t \leqslant 0$;

$\left(\mathrm{f}_{2}\right)$ for $f_{0}, f_{\infty}<\infty$, the limits

$$
\lim _{t \rightarrow 0^{+}} \frac{f(x, t)}{t}=f_{0}, \quad \lim _{t \rightarrow \infty} \frac{f(x, t)}{t^{p-1}}=f_{\infty}
$$

exist uniformly for $x \in \bar{\Omega}$.

The asymptotic behaviors of $f$ near zero and infinity lead us to define

$$
\begin{aligned}
& \lambda_{1}=\inf \left\{\int_{\Omega}|\nabla u|^{2}: u \in H_{0}^{1}(\Omega), \int_{\Omega}|u|^{2}=1\right\}, \\
& \mu_{1}=\inf \left\{\int_{\Omega}|\nabla u|^{p}: u \in W_{0}^{1, p}(\Omega), \int_{\Omega}|u|^{p}=1\right\},
\end{aligned}
$$

where $H_{0}^{1}(\Omega)$ and $W_{0}^{1, p}(\Omega)$ are the usual Sobolev spaces defined as the completion of $C_{0}^{\infty}(\Omega)$ with respect to the norms $\|u\|_{2}=\left(\int_{\Omega}|\nabla u|^{2}\right)^{1 / 2}$ and $\|u\|_{p}=\left(\int_{\Omega}|\nabla u|^{p}\right)^{1 / p}$, respectively. Then it is well known that $\mu_{1}$ is the first eigenvalue of the nonlinear eigenvalue problem

$$
\begin{gathered}
-\Delta_{p} \phi=\mu|\phi|^{p-2} \phi, \quad \text { in } \Omega, \\
\phi=0, \quad \text { on } \partial \Omega .
\end{gathered}
$$

Moreover $\mu_{1}$ is a simple eigenvalue of (4), the associated eigenfunction $\phi_{1}$ can be chosen as positive in $\Omega$, and any eigenfunction corresponding to an eigenvalue larger than $\mu_{1}$ must change sign. The reader is referred to [1-3] for details.

By a solution $u$ of (1), we mean that $u$ solves (1) in the weak sense; that is, $u$ satisfies

$$
\begin{array}{r}
\int_{\Omega} \nabla u \cdot \nabla v+\int_{\Omega}|\nabla u|^{p-2} \nabla u \cdot \nabla v= \\
\int_{\Omega} f(x, u) v \\
v \in W_{0}^{1, p}(\Omega) .
\end{array}
$$

Moreover, by a positive solution $u$ of (1), we mean that $u$ is a weak solution of (1), $u \neq 0$ and $u(x) \geqslant 0$ for $x \in \Omega$.

Our main result is the following theorem.

Theorem 1. Suppose that $f$ satisfies $\left(f_{1}\right)$ and $\left(f_{2}\right)$ with $f_{0}<$ $\lambda_{1}, f_{\infty}>\mu_{1}$. Then (1) has a positive solution. 
Assume that $f=g+h$. Equation (1) can be viewed as combination of the following equations:

$$
\begin{aligned}
-\Delta u & =g(x, u), \quad \text { in } \Omega, \\
u & =0, \quad \text { on } \partial \Omega, \\
-\Delta_{p} u & =h(x, u), \quad \text { in } \Omega, \\
u & =0, \quad \text { on } \partial \Omega .
\end{aligned}
$$

In the last decade or so, there was an extensive effort in studying the existence of solutions of (6); see [4-8].

Before concluding this section, we recall a theorem from [9], which will be used to prove our main theorem in this paper.

Theorem 2. Let $(E,\|\cdot\|)$ be a Banach space and $I \subset \mathbb{R}_{+}$an interval. Consider the family of $C^{1}$ functionals on $E$,

$$
J_{v}(u)=S(u)-v T(u), \quad v \in I
$$

with $J_{\nu}(0)=0, v \in I, T$ nonnegative and either $S(u) \rightarrow \infty$ or $T(u) \rightarrow \infty$ as $\|u\| \rightarrow \infty$. For any $v \in I$, we set

$$
\Gamma_{\nu}=\left\{\gamma \in C([0,1], E): \gamma(0)=0, J_{\nu}(\gamma(1))<0\right\} .
$$

If for every $\nu \in I$ the set $\Gamma_{v}$ is nonempty and

$$
c_{v}=\inf _{\gamma \in \Gamma_{\nu}} \max _{t \in[0,1]} J_{\nu}(\gamma(t))>0,
$$

then for almost every $v \in I$ there exists a sequence $\left\{u_{n}^{\nu}\right\} \subset E$ such that

(i) $\left\{u_{n}^{\nu}\right\}$ is bounded;

(ii) $J_{\nu}\left(u_{n}^{v}\right) \rightarrow c_{\nu}$ as $n \rightarrow \infty$;

(iii) $J_{v}^{\prime}\left(u_{n}^{\nu}\right) \rightarrow 0$ in the dual $E^{*}$ as $n \rightarrow \infty$.

Throughout this paper, we denote by $X$ the Sobolev space $W_{0}^{1, p}(\Omega)$ with the norm $\|u\|_{X}=\|u\|_{p}$, by $X^{*}$ the duality space of $X$, by $\rightarrow$ the weak convergence in $X$, and by $\langle\cdot, \cdot\rangle$ the duality pairing between $X^{*}$ and $X$. The letters $C_{1}, C_{2}, \ldots$ will denote various positive constants whose exact values are not essential to the analysis of the problem. Let $P=\{u \in X: u(x) \geqslant 0$, a.e. $x \in \Omega\}$ and $p^{*}=N p /(N-p)$ if $p<N$ or $\infty$ if $p \geqslant N$.

\section{Proof of Theorem 1}

In this section, we always assume $\left(\mathrm{f}_{1}\right)$ and $\left(\mathrm{f}_{2}\right)$ hold with $f_{0}<$ $\lambda_{1}$ and $f_{\infty}>\mu_{1}$. Hence, there exist $\varepsilon_{1}>0, C_{\varepsilon_{1}}>0$ and $q \epsilon$ $\left(p, p^{*}\right)$ such that

$$
\begin{gathered}
F(x, t) \geqslant \frac{1}{p}\left(\mu_{1}+\varepsilon_{1}\right) t^{p}-C_{\varepsilon_{1}}, \quad x \in \bar{\Omega}, t \geqslant 0, \\
F(x, t) \leqslant \frac{1}{2}\left(1-\varepsilon_{1}\right) \lambda_{1} t^{2}+C_{\varepsilon_{1}} t^{q}, \quad x \in \bar{\Omega}, t \in \mathbb{R},
\end{gathered}
$$

where $F(x, t)=\int_{0}^{t} f(x, s) d s$. In the following, we utilize Theorem 2 to complete the proof of Theorem 1 . In the setting of Theorem 2 we have $E=X, I=[\delta, 1]$ with $\mu_{1} /\left(\mu_{1}+\varepsilon_{1}\right)<$ $\delta<1$, and

$$
\begin{gathered}
S(u)=\frac{1}{2}\|u\|_{2}^{2}+\frac{1}{p}\|u\|_{X}^{p}, \quad T(u)=\int_{\Omega} F(x, u), \\
J_{\nu}(u)=\frac{1}{2}\|u\|_{2}^{2}+\frac{1}{p}\|u\|_{X}^{p}-v \int_{\Omega} F(x, u), \quad u \in X, v \in I .
\end{gathered}
$$

It is easy to verify that

$$
\begin{array}{r}
\left\langle J_{v}^{\prime}(u), v\right\rangle \\
=\int_{\Omega} \nabla u \cdot \nabla v+\int_{\Omega}|\nabla u|^{p-2} \nabla u \cdot \nabla v-v \int_{\Omega} f(x, u) v, \\
u, v \in X, \quad v \in I .
\end{array}
$$

Firstly, we show that $J_{v}$ satisfies the conditions of Theorem 2 by proving several lemmas.

Lemma 3. $\Gamma_{\nu} \neq \emptyset$ for any $v \in I$.

Proof. Let $\phi_{1}>0$ be a $\mu_{1}$-eigenfunction. For $t>0$, we have by (10) that

$$
\begin{aligned}
J_{\nu}\left(t \phi_{1}\right)= & \frac{1}{2} t^{2}\left\|\phi_{1}\right\|_{2}^{2}+\frac{1}{p} t^{p}\left\|\phi_{1}\right\|_{X}^{p}-v \int_{\Omega} F\left(x, t \phi_{1}\right) \\
\leqslant & \frac{1}{2} t^{2}\left\|\phi_{1}\right\|_{2}^{2}+\frac{1}{p} \mu_{1} t^{p} \int_{\Omega}\left|\phi_{1}\right|^{p} \\
& -\frac{1}{p}\left(\mu_{1}+\varepsilon_{1}\right) \delta t^{p} \int_{\Omega}\left|\phi_{1}\right|^{p}+C_{1} \\
= & \frac{1}{2} t^{2}\left\|\phi_{1}\right\|_{2}^{2}-\frac{1}{p} C_{2} t^{p} \int_{\Omega}\left|\phi_{1}\right|^{p}+C_{1},
\end{aligned}
$$

where $C_{2}=\left(\mu_{1}+\varepsilon_{1}\right) \delta-\mu_{1}$. Noting that $C_{2}>0$, we can choose $t_{0}>0$ large enough so that $J_{v}\left(t_{0} \phi_{1}\right)<0$, where $t_{0}$ is independent of $\nu \in I$. The proof is completed.

Lemma 4. There exists a constant $c>0$ such that $c_{v} \geqslant c$ for any $\nu \in I$.

Proof. For any $u \in X$, it follows from (11) that

$$
\begin{aligned}
J_{v}(u) & =\frac{1}{2}\|u\|_{2}^{2}+\frac{1}{p}\|u\|_{X}^{p}-v \int_{\Omega} F(x, u) \\
& \geqslant \frac{1}{2}\|u\|_{2}^{2}+\frac{1}{p}\|u\|_{X}^{p}-\frac{1}{2}\left(1-\varepsilon_{1}\right) \lambda_{1} \int_{\Omega}|u|^{2}-C_{\varepsilon_{1}} \int_{\Omega}|u|^{q} \\
& \geqslant \frac{1}{2}\|u\|_{2}^{2}+\frac{1}{p}\|u\|_{X}^{p}-\frac{1}{2}\left(1-\varepsilon_{1}\right)\|u\|_{2}^{2}-C_{\varepsilon_{1}} \int_{\Omega}|u|^{q} \\
& \geqslant \frac{1}{p}\|u\|_{X}^{p}-C_{\varepsilon_{1}} \int_{\Omega}|u|^{q} .
\end{aligned}
$$

By Sobolev's embedding theorem, we conclude that there exist $\rho>0$ and $c>0$ such that $J_{\nu}(u)>0$ for $\|u\| \in(0, \rho]$ and

$$
J_{v}(u) \geqslant c, \quad\|u\|_{X}=\rho .
$$


Fix $\nu \in I$ and $\gamma \in \Gamma_{\nu}$. By the definition of $\Gamma_{\nu}$, we have that $\|\gamma(1)\|>\rho$. Hence, there exists $t_{\gamma} \in(0,1)$ such that $\left\|\gamma\left(t_{\gamma}\right)\right\|=$ $\rho$. So

$$
c_{\nu}=\inf _{\gamma \in \Gamma_{\nu}} \max _{t \in[0,1]} J_{\nu}(\gamma(t)) \geqslant \inf _{\gamma \in \Gamma_{\nu}} J_{\nu}\left(\gamma\left(t_{\gamma}\right)\right) \geqslant c .
$$

The proof is completed.

Lemma 5. For any $v \in I$, if $\left\{u_{n}\right\}$ is bounded and $J_{v}^{\prime}\left(u_{n}\right) \rightarrow 0$ in $X^{*}$ as $n \rightarrow \infty$, then $\left\{u_{n}\right\}$ admits a convergent subsequence.

Proof. Given $v \in I$, assume that $\left\{u_{n}\right\}$ is bounded, $J_{\nu}^{\prime}\left(u_{n}\right) \rightarrow$ 0 in $X^{*}$ as $n \rightarrow \infty$. By extracting a subsequence, we may suppose that there exists $u \in X$ such that as $n \rightarrow \infty$

$$
\begin{gathered}
u_{n} \rightarrow u \quad \text { in } X, \\
u_{n} \rightarrow u \quad \text { in } L^{s}(\Omega), \quad s \in\left[1, p^{*}\right) .
\end{gathered}
$$

It follows from $\left(\mathrm{f}_{1}\right)$ and $\left(\mathrm{f}_{2}\right)$ that there exist $C_{1}, C_{2}>0$ such that

$$
f(x, t) \leqslant C_{1}|t|+C_{2}|t|^{p-1}, \quad x \in \bar{\Omega}, t \in \mathbb{R} .
$$

Hence, by Hölder's inequality and Sobolev's embedding theorem, we have

$$
\begin{aligned}
& \left|\int_{\Omega} f\left(x, u_{n}\right)\left(u_{n}-u\right)\right| \\
& \leqslant C_{1} \int_{\Omega}\left|u_{n}\right|\left|u_{n}-u\right|+C_{2} \int_{\Omega}\left|u_{n}\right|^{p-1}\left|u_{n}-u\right| \\
& \leqslant C_{1}\left(\int_{\Omega}\left|u_{n}\right|^{2}\right)^{1 / 2}\left(\int_{\Omega}\left|u_{n}-u\right|^{2}\right)^{1 / 2} \\
& \quad+C_{2}\left(\int_{\Omega}\left|u_{n}\right|^{p}\right)^{(p-1) / p}\left(\int_{\Omega}\left|u_{n}-u\right|^{p}\right)^{1 / p} \\
& \leqslant C_{3}\left(\int_{\Omega}\left|u_{n}-u\right|^{2}\right)^{1 / 2}+C_{4}\left(\int_{\Omega}\left|u_{n}-u\right|^{p}\right)^{1 / p} \longrightarrow 0
\end{aligned}
$$$$
n \longrightarrow \infty \text {. }
$$

Similarly, we have

$$
\left|\int_{\Omega} f(x, u)\left(u_{n}-u\right)\right| \longrightarrow 0, \quad n \longrightarrow \infty .
$$

Noting that

$$
\begin{aligned}
\left\langle J_{v}^{\prime}\left(u_{n}\right)-J_{v}^{\prime}(u), u_{n}-u\right\rangle & \\
= & \left\langle J_{\nu}^{\prime}\left(u_{n}\right), u_{n}-u\right\rangle-\left\langle J_{v}^{\prime}(u), u_{n}-u\right\rangle \\
= & \int_{\Omega} \nabla u_{n} \cdot \nabla\left(u_{n}-u\right)+\int_{\Omega}\left|\nabla u_{n}\right|^{p-2} \nabla u_{n} \cdot \nabla\left(u_{n}-u\right) \\
& -v \int_{\Omega} f\left(x, u_{n}\right)\left(u_{n}-u\right)-\int_{\Omega} \nabla u \cdot \nabla\left(u_{n}-u\right) \\
& -\int_{\Omega}|\nabla u|^{p-2} \nabla u \cdot \nabla\left(u_{n}-u\right)+v \int_{\Omega} f(x, u)\left(u_{n}-u\right) \\
= & \int_{\Omega}\left|\nabla\left(u_{n}-u\right)\right|^{2} \\
& +\int_{\Omega}\left(\left|\nabla u_{n}\right|^{p-2} \nabla u_{n}-|\nabla u|^{p-2} \nabla u\right) \cdot \nabla\left(u_{n}-u\right) \\
& -v \int_{\Omega} f\left(x, u_{n}\right)\left(u_{n}-u\right)+v \int_{\Omega} f(x, u)\left(u_{n}-u\right)
\end{aligned}
$$

and the inequality deduced from an inequality in Appendix of [3],

$$
\begin{gathered}
\int_{\Omega}\left(\left|\nabla u_{n}\right|^{p-2} \nabla u_{n}-|\nabla u|^{p-2} \nabla u\right) \cdot \nabla\left(u_{n}-u\right) \\
\geqslant \frac{2}{p\left(2^{p-1}-1\right)} \int_{\Omega}\left|\nabla\left(u_{n}-u\right)\right|^{p},
\end{gathered}
$$

it follows from (20) and (21) that

$$
\begin{aligned}
\frac{2}{p\left(2^{p-1}-1\right)} \int_{\Omega}\left|\nabla\left(u_{n}-u\right)\right|^{p} \leqslant & \left\langle J_{\nu}^{\prime}\left(u_{n}\right)-J_{v}^{\prime}(u), u_{n}-u\right\rangle \\
& +v \int_{\Omega} f\left(x, u_{n}\right)\left(u_{n}-u\right) \\
& -v \int_{\Omega} f(x, u)\left(u_{n}-u\right) \\
& \longrightarrow 0, \quad n \longrightarrow \infty
\end{aligned}
$$

where we have used the fact that

$$
\left\langle J_{v}^{\prime}\left(u_{n}\right)-J_{v}^{\prime}(u), u_{n}-u\right\rangle \longrightarrow 0, \quad n \longrightarrow \infty .
$$

Hence $u_{n} \rightarrow u$ in $X$. The proof is completed.

Lemma 6. There exists a sequence $\left\{v_{n}\right\} \subset I$ with $\nu_{n} \rightarrow 1^{-}$as $n \rightarrow \infty$ and $\left\{u_{v_{n}}\right\} \subset X$ such that $J_{v_{n}}\left(u_{v_{n}}\right)=c_{v_{n}}, J_{v_{n}}^{\prime}\left(u_{v_{n}}\right)=0$.

Proof. We only need to show that for almost every $\nu \in I$ there exists $u^{\nu} \in X$ such that $J_{\nu}\left(u^{\nu}\right)=c_{v}$ and $J_{\nu}^{\prime}\left(u^{\nu}\right)=0$. By Theorem 2, for almost each $v \in I$, there exists a bounded sequence $\left\{u_{n}^{\nu}\right\} \subset X$ such that

$$
J_{\nu}\left(u_{n}^{\nu}\right) \longrightarrow c_{v}, \quad J_{v}^{\prime}\left(u_{n}^{\nu}\right) \longrightarrow 0, \quad n \longrightarrow \infty .
$$


By Lemma 5, we may assume that $u_{n}^{v} \rightarrow u^{v}$ in $X$ as $n \rightarrow \infty$. Then the continuity of $J_{v}$ and $J_{v}^{\prime}$ implies that $J_{v}\left(u^{v}\right)=c_{v}$ and $J_{v}^{\prime}\left(u^{v}\right)=0$. The proof is completed.

Define $\langle L u, v\rangle=\int_{\Omega} f(x, u) v,\langle K u, v\rangle=\int_{\Omega}|u|^{p-2} u v$, $u, v \in X$. Then we have the following.

Lemma 7. Supposing $\left(f_{1}\right)$ and $\left(f_{2}\right)$ hold, then

$$
\lim _{\|u\|_{X} \rightarrow \infty, u \in P} \frac{L u-f_{\infty} K u}{\|u\|_{X}^{p-1}}=0 .
$$

Proof. By $\left(\mathrm{f}_{1}\right),\left(\mathrm{f}_{2}\right)$, for every $\varepsilon>0$, there is a constant $C_{\varepsilon}>0$ such that

$$
\left|f(x, t)-f_{\infty} t^{p-1}\right| \leqslant C_{\varepsilon}+\varepsilon t^{p-1}, \quad x \in \bar{\Omega}, t \geqslant 0 .
$$

For $u \in P \backslash\{0\}$, letting $w=u /\|u\|_{X}$, by Hölder's inequality and Sobolev's embedding theorem, we have

$$
\begin{aligned}
& \sup _{\|v\|_{X} \leqslant 1}\left|\left\langle\frac{L u-f_{\infty} K u}{\|u\|_{X}^{p-1}}, v\right\rangle\right| \\
& \quad \leqslant \sup _{\|v\|_{X} \leqslant 1} \int_{\Omega} \frac{\left|f(x, u)-f_{\infty} u^{p-1}\right|}{\|u\|_{X}^{p-1}}|v| \\
& \leqslant \sup _{\|\nu\|_{X} \leqslant 1} \int_{\Omega} C_{\varepsilon}\|u\|_{X}^{-(p-1)}|v|+\varepsilon w^{p-1}|v| \\
& \leqslant C_{6}\|u\|_{X}^{-(p-1)}+\varepsilon C_{5},
\end{aligned}
$$

where $C_{5}$ is independent of $\varepsilon$. The proof is completed.

Proof of Theorem 1. By Lemma 6, there exists a sequence $\left\{v_{n}\right\} \subset I$ with $v_{n} \rightarrow 1^{-}$as $n \rightarrow \infty$ and $\left\{u_{v_{n}}\right\} \subset X$ such that

$$
J_{v_{n}}\left(u_{v_{n}}\right)=c_{v_{n}}, \quad J_{v_{n}}^{\prime}\left(u_{v_{n}}\right)=0 .
$$

By Lemma 4 and (30), we have $c_{v_{n}} \geqslant c>0$ and $\left\langle J_{v_{n}}^{\prime}\left(u_{v_{n}}\right), u_{v_{n}}^{-}\right\rangle=0$. Hence $u_{v_{n}} \in P \backslash\{0\}$. In the following, we first claim that $\left\{u_{v_{n}}\right\}$ is bounded in $X$. Suppose by contradiction that $\lim _{n \rightarrow \infty}\left\|u_{v_{n}}\right\|_{X}=\infty$. Let $w_{n}=u_{v_{n}} /\left\|u_{v_{n}}\right\|_{X}$. Hence, we have, for $v \in X$,

$$
\begin{aligned}
& \frac{1}{\left\|u_{v_{n}}\right\|_{X}^{p-2}} \int_{\Omega} \nabla w_{n} \nabla v+\int_{\Omega}\left|\nabla w_{n}\right|^{p-2} \nabla w_{n} \nabla v \\
& \quad=v_{n} f_{\infty} \int_{\Omega} w_{n}^{p-1} v+v_{n} \int_{\Omega} \frac{f\left(x, u_{v_{n}}\right)-f_{\infty} u_{v_{n}}^{p-1}}{\left\|u_{v_{n}}\right\|_{X}^{p-1}} v .
\end{aligned}
$$

Since $\left\{w_{n}\right\}$ is bounded in $X$, we may assume that $w_{n} \rightarrow w_{0} \in$ $P \subset X, w_{n} \rightarrow w_{0}$ in $L^{p}(\Omega)$ and $w_{n}(x) \rightarrow w_{0}(x)$ a.e. on $\Omega$ as $n \rightarrow \infty$. Letting $v=w_{n}-w_{0}$ in (31) and $n \rightarrow \infty$, we get

$$
\lim _{n \rightarrow \infty} \int_{\Omega}\left|\nabla w_{n}\right|^{p-2} \nabla w_{n} \cdot \nabla\left(w_{n}-w_{0}\right)=0 .
$$

It follows from [5, Theorem 10] that $w_{n} \rightarrow w_{0}$ in $X$ as $n \rightarrow$ $\infty$. Passing to limit $n \rightarrow \infty$ in (31), we obtain by Lemma 7 that

$$
\int_{\Omega}\left|\nabla w_{0}\right|^{p-2} \nabla w_{0} \cdot \nabla v=f_{\infty} \int_{\Omega} w_{0}^{p-1} v, \quad v \in X .
$$

From (33) and the fact that $\left\|w_{0}\right\|_{X}=1$, we know that $f_{\infty}=\mu_{1}$, which contradicts the assumption $f_{\infty}>\mu_{1}$. Since $\nu_{n} \rightarrow 1^{-}$, we can show that

$$
J_{1}^{\prime}\left(u_{v_{n}}\right) \longrightarrow 0 \quad \text { in } X^{*}, \quad n \longrightarrow \infty .
$$

In fact, for any $v \in X$, it follows from (19), Hölder's inequality, and Sobolev's embedding theorem that

$$
\begin{aligned}
\left|\int_{\Omega} f\left(x, u_{v_{n}}\right) v\right| & \leqslant C_{1} \int_{\Omega}\left|u_{v_{n}}\right||v|+C_{2} \int_{\Omega}\left|u_{v_{n}}\right|^{p-1}|v| \\
& \leqslant C_{7}\|v\|_{X} .
\end{aligned}
$$

Furthermore, (30) implies that

$$
\begin{array}{r}
\left\langle J_{1}^{\prime}\left(u_{v_{n}}\right), v\right\rangle+\left(1-v_{n}\right) \int_{\Omega} f\left(x, u_{v_{n}}\right) v=\left\langle J_{v_{n}}^{\prime}\left(u_{v_{n}}\right), v\right\rangle=0, \\
v \in X .
\end{array}
$$

Hence, $J_{1}^{\prime}\left(u_{v_{n}}\right) \rightarrow 0$ in $X^{*}$ as $n \rightarrow \infty$. By Lemma $5,\left\{u_{v_{n}}\right\}$ has a convergent subsequence. Without loss of generality, we may assume that $u_{v_{n}} \rightarrow u$ as $n \rightarrow \infty$. According to Lemma 4, (30) and

$$
\left|\int_{\Omega} F\left(x, u_{v_{n}}\right)\right| \leqslant C_{8}
$$

we have

$$
\begin{gathered}
J_{1}(u)=\lim _{n \rightarrow \infty} J_{1}\left(u_{v_{n}}\right)=\lim _{n \rightarrow \infty} J_{v_{n}}\left(u_{v_{n}}\right) \geqslant c>0, \\
J_{1}^{\prime}(u)=\lim _{n \rightarrow \infty} J_{1}^{\prime}\left(u_{v_{n}}\right)=0 .
\end{gathered}
$$

The standard process shows that $u$ is a positive solution to (1). The proof is completed.

\section{Conflict of Interests}

The authors declare that there is no conflict of interests regarding the publication of this paper.

\section{Acknowledgments}

This paper is partially supported by National Natural Science Foundation of China (Grant nos. 11071149, 11301313) and Science Council of Shanxi Province (2012011004-2, 20130210014, and 2014021009-1).

\section{References}

[1] A. Anane, "Simplicité et isolation de la première valeur propre du p-laplacien avec poids," Comptes Rendus de l'Académie des Sciences: Mathématique, vol. 305, no. 16, pp. 725-728, 1987. 
[2] B. Kawohl and P. Lindqvist, "Positive eigenfunctions for the $p$ Laplace operator revisited," Analysis, vol. 26, no. 4, pp. 545-550, 2006.

[3] P. Lindqvist, "On the equation $\operatorname{div}\left(\left|\nabla_{u}\right|^{p-2} \nabla_{u}\right)+\lambda|u|^{p-2} u=0$," Proceedings of the American Mathematical Society, vol. 109, no. 1, pp. 157-164, 1990.

[4] K.-C. Chang, Infinite Dimensional Morse Theory and Multiple Solution Problems, vol. 6 of Progress in Nonlinear Differential Equations and Their Applications, Birkhauser, Boston, Mass, USA, 1993.

[5] G. Dinca, P. Jebelean, and J. Mawhin, "Variational and topological methods for Dirichlet problems with p-Laplacian," Portugaliae Mathematica, vol. 58, no. 3, pp. 339-378, 2001.

[6] Y. Huang and H.-S. Zhou, "Positive solution for $-\triangle_{p} u=$ $f(x, u)$ with $f(x, u)$ with $f(x, u)$ growing as $u^{p-1}$ at infinity," Applied Mathematics Letters, vol. 17, no. 8, pp. 881-887, 2004.

[7] P. H. Rabinowitz, "Minimax methods in critical point theory with applications to differential equations," in Proceedings of the Conference Board of the Mathematic al Sciences, vol. 65 of CBMS Regional Conference Series in Mathematics, American Mathematical Society, Washington, DC, USA, 1986.

[8] M. Willem, Progress in Nonlinear Differential Equations and Their Applications, Birkhäauser, Boston, Mass, USA, 1996.

[9] L. Jeanjean, "Local conditions insuring bifurcation from the continuous spectrum," Mathematische Zeitschrift, vol. 232, no. 4, pp. 651-664, 1999. 


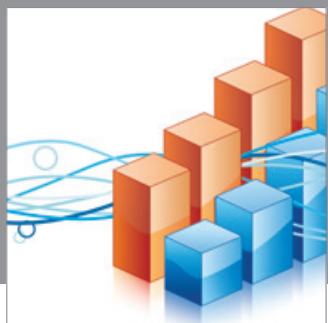

Advances in

Operations Research

mansans

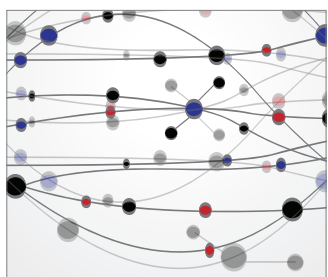

The Scientific World Journal
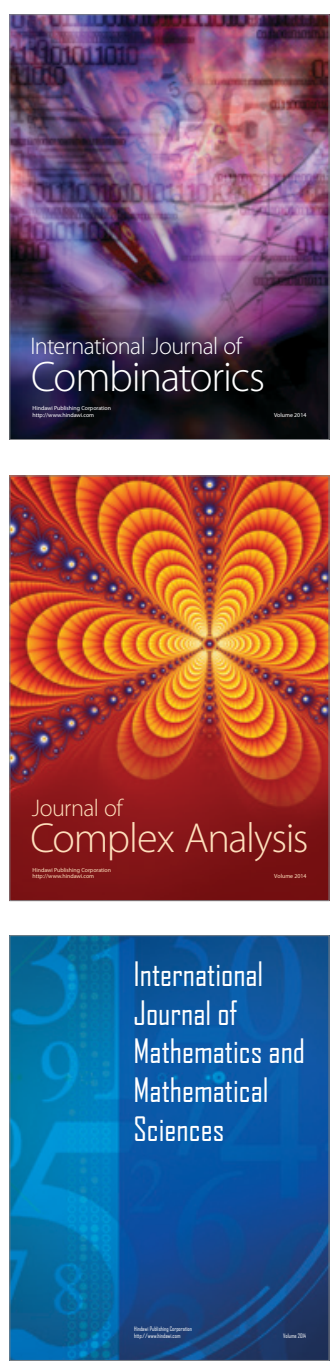
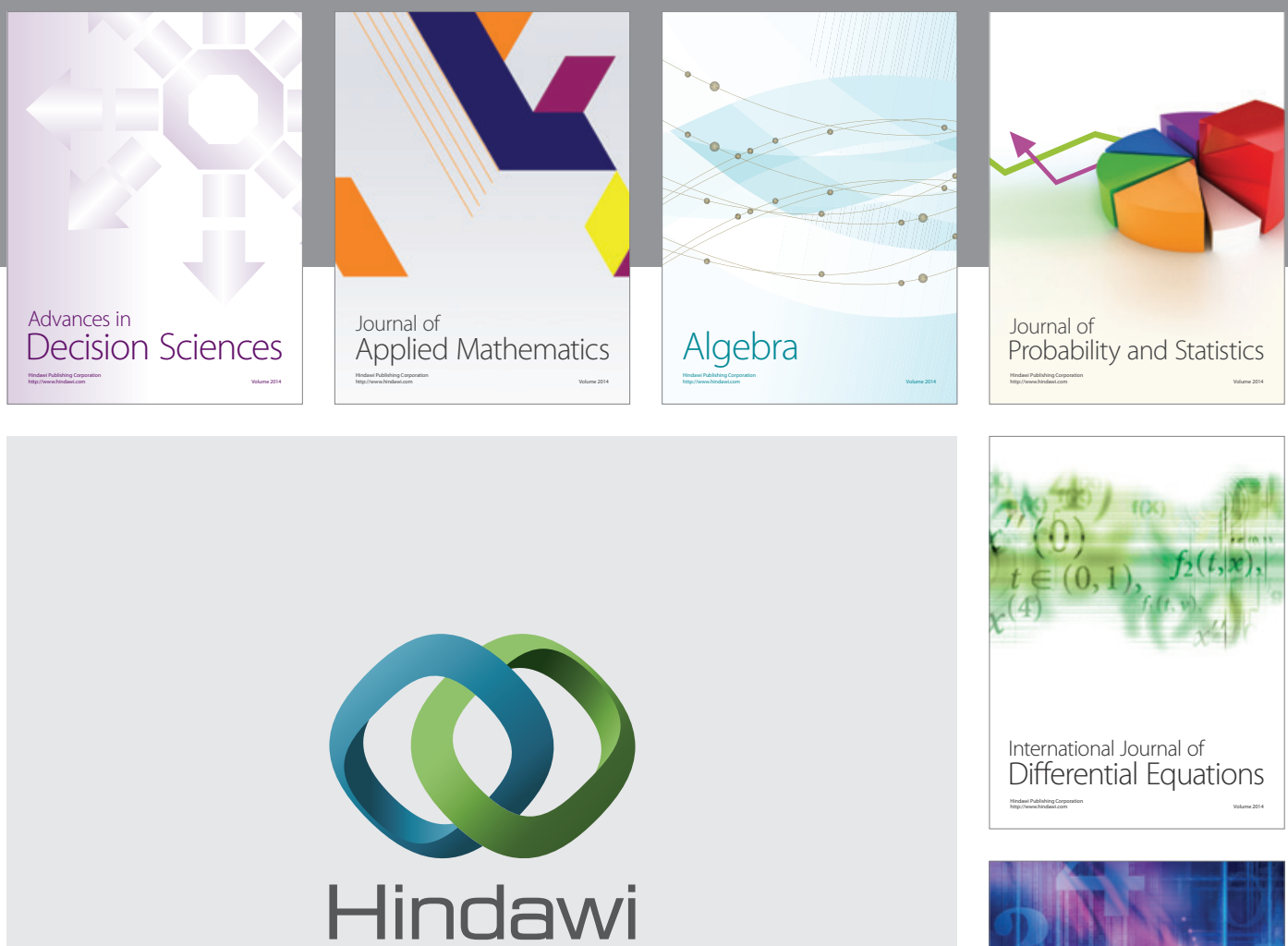

Submit your manuscripts at http://www.hindawi.com
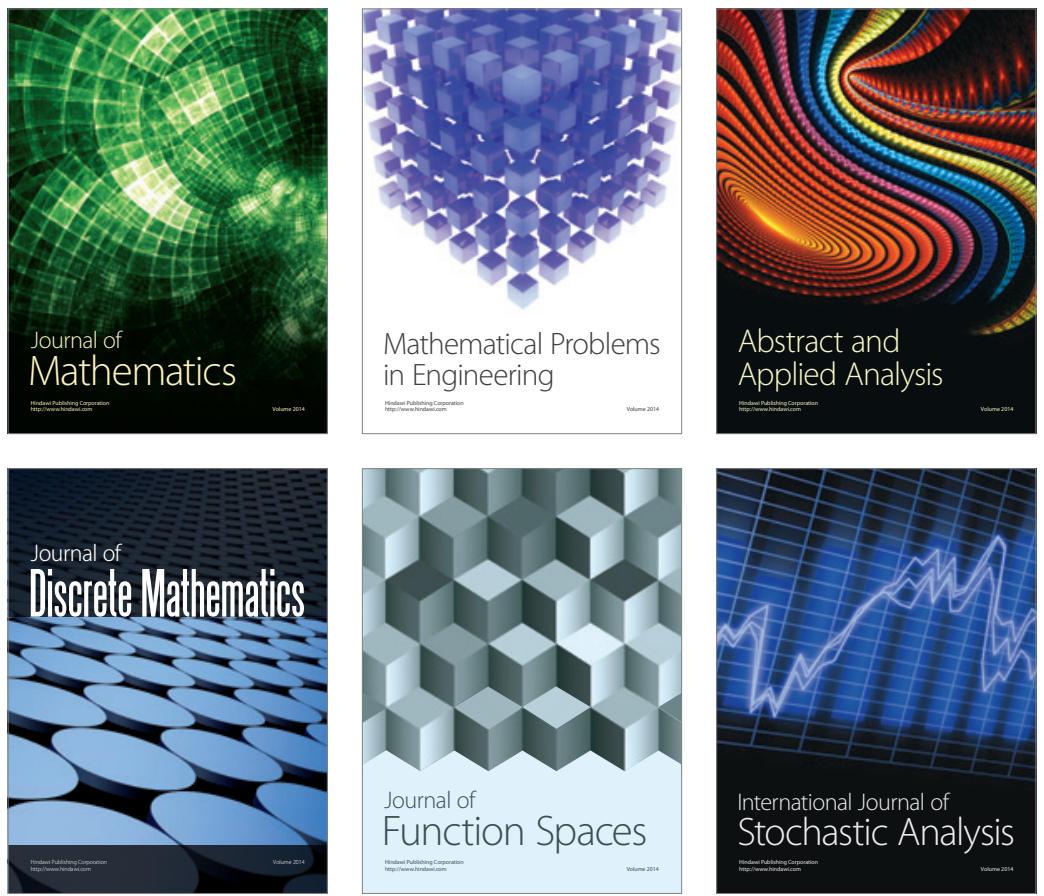

Journal of

Function Spaces

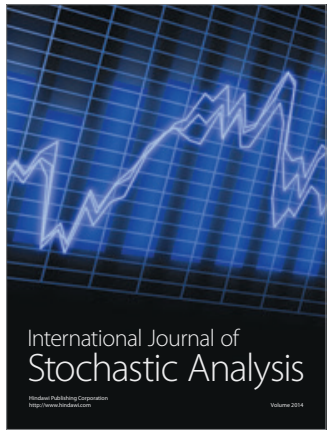

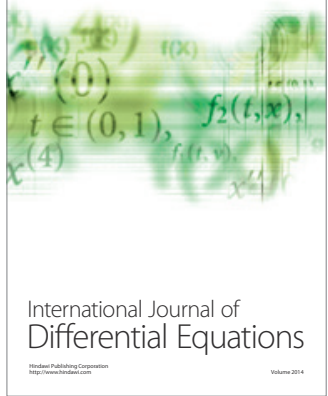
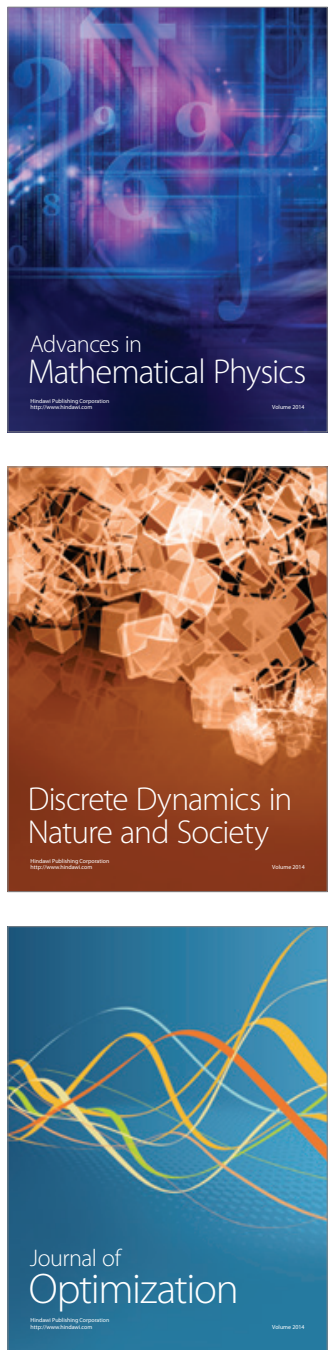